\title{
Conservation laws in anisotropic elasticity II. Extension and application to thermoelasticity $\dagger$
}

\author{
B Y C.-S. YEH ${ }^{1}$, Y.-C. $\mathrm{SH} \mathrm{U}^{2}$ A N K K.-C. W U ${ }^{3}$ \\ ${ }^{1}$ Department of Civil Engineering and Institute of Applied Mechanics, \\ ${ }^{2}$ Department of Civil Engineering and ${ }^{3}$ Institute of Applied Mechanics, \\ National Taiwan University, Taipei 106, Taiwan, R.O.C.
}

The conservation laws in anisotropic elasticity developed in an accompanying paper are extended to include steady-state thermal elasticity. The conservation laws proposed in this paper lead to integrals that do not contain area integration and are path-independent. In addition to the extended $J$ - and $M$-integrals of J. K. Knowels and E. Sternberg, also derived are path-independent contour integrals that yield directly the stress intensity factors when evaluated over contours enclosing a crack. The path-independent integral representations of the stress intensity factors are used to obtain closed form solutions for a finite crack in an unbounded thermoelastic medium subject to arbitrary thermal conditions on the crack faces.

\section{Introduction}

In this paper the conservation laws for anisotropic thermoelastic materials are considered. Wilson \& Yu (1979) and Kishimoto et al. (1980) have derived conservation laws for thermal stress crack problems. The expressions of the conservation laws, however, contain area integral terms. The presence of these area integral terms is undesirable in linear elastic fracture mechanics in that the energy release rate or stress intensity factors associated with a crack tip can not be extracted from far-field data alone using the conservation laws. Gurtin (1979) has derived a conservation law, valid in both two- or three-dimensional space, for steadystate linear isotropic thermoelasticity. The conservation law obtained by Gurtin is in the form of a contour integral which is path-independent under certain restrictions which are not usually met. Three additional material conservation laws for the same problems in the two-dimensional space have been proposed by Kuo \& Riccardella (1987) by introducing a conjugate temperature variable. With the introduction of the conjugate temperature, the conservation laws given by Kuo \& Riccardella are expressed in terms of contour integrals which are pathindependent without any restrictions.

The aim here is to derive anisotropic steady-state thermoelastic conservation laws which yield contour integrals that are path-independent. The development

$\dagger$ This paper was produced from the authors' disk by using the TEX typesetting system.

Proc. R. Soc. Lond. A (1993) 443, 153-161

(C) 1993 The Royal Society

Printed in Great Britain 
is based on the ideas that a thermoelastic state can be regarded as the superposition of an elastic state in the absence of body forces and another elastic state due to effective body forces resulting from non-uniform temperature distribution (Clements 1973; Wu 1984; Hwu 1990) and that the conservation laws constructed with respect to the state without body forces result in path-independent integrals. It is assumed that the temperature is finite at the crack tip so that the singular crack tip fields do not alter with non-uniform temperature distribution. Under this assumption, the conservation laws proposed here possess the same physical meanings as their counterparts for isothermal elasticity. Although the conservation laws derived in this work do not include area integrals, use of the conservation laws to determine the energy release rates or stress intensity factors involves integration along contours including the crack faces in general. In addition to the extensions of the familiar $J$ - and $M$-integrals, also derived are path-independent contour integrals that yield directly the stress intensity factors when evaluated over contours enclosing a crack. The path-independent integral representations of the stress intensity factors are used to obtain closed form solutions for a finite crack in an unbounded thermoelastic medium subject to arbitrary thermal conditions on the crack faces.

\section{Basic equations of thermoelasticity}

Basic equations and an extended formalism due to Hwu (1990) for anisotropic thermal elasticity are introduced. Hereafter, summation over repeated indices is implied and a comma stands for partial differentiation; bold symbols represent vectors, tensors or matrices.

With respect to a fixed rectangular cartesian coordinates, the basic equations of steady-state linear homogeneous thermoelasticity in the absence of body forces and heat supplies consist of (Nowacki 1962):

(i) equations of equilibrium

(ii) energy equation

$$
\sigma_{i j, j}=0
$$

(iii) constitutive equations

$$
q_{j, j}=0
$$

$$
\begin{aligned}
\sigma_{i j} & =C_{i j k l} u_{k, l}-\beta_{i j} T, \\
q_{j} & =-k_{i j} T_{, i} .
\end{aligned}
$$

in which $\boldsymbol{u}, T, \boldsymbol{\sigma}$, and $\boldsymbol{q}$ are the displacement, temperature, stress, and heat flux respectively; whereas $\boldsymbol{C}, \boldsymbol{\beta}$ and $\boldsymbol{k}$ are the elastic constant of the generalized Hooke's law, thermal modulus and heat conductivity with the following symmetry relations

$$
\left.\begin{array}{c}
C_{i j k l}=C_{k l i j}=C_{j i k l}=C_{i j l k}, \\
\beta_{i j}=\beta_{j i}, \quad k_{i j}=k_{j i} .
\end{array}\right\}
$$

Substituting (2.3) and (2.4) into (2.1) and (2.2), we arrive at the governing equations in terms of displacements and temperature

$$
\begin{aligned}
C_{i j k l} u_{k, l j}-\beta_{i j} T_{, j} & =0, \\
k_{i j} T_{, i j} & =0 .
\end{aligned}
$$

Proc. R. Soc. Lond. A (1993) 
As (2.6) and (2.7) are uncoupled, to determine the displacement field the temperature field must be solved in advance with the appropriate thermal boundary conditions

$$
q_{j} n_{j}=q_{o} \quad \text { on } \quad \partial \Omega_{q}, \quad T=T_{o} \quad \text { on } \quad \partial \Omega_{T},
$$

where the subscript $o$ denotes the specified functions on the boundaries.

We first consider the two-dimensional steady-state heat conduction problem where the temperature distribution is independent of $x_{3}$. Since (2.7) is similar in form as that for antiplane deformation in anisotropic materials, the general solutions to the heat conduction problem can be obtained from those for antiplane deformation problem given by Lehknitskii (1963). The solutions for $T$ are expressed as

$$
T=2 \operatorname{Re}\left[g^{\prime}\left(z_{t}\right)\right], \quad z_{t}=x_{1}+\tau x_{2},
$$

where $g^{\prime}\left(z_{t}\right)$ is an arbitrary function and prime stands for differentiation with respect to its argument; Re denotes the real part; the thermal eigenvalue $\tau$ is the solution of

$$
k_{22} \tau^{2}+2 k_{12} \tau+k_{11}=0
$$

Here, the assumption of positive definiteness of the heat conduction constants $k_{i j}$ is adopted such that the thermal eigenvalue $\tau$ can not be a real number in (2.10). By defining an heat function $Q$ such that

$$
\partial Q / \partial x_{2}=-q_{1}, \quad \partial Q / \partial x_{1}=q_{2} .
$$

The complex function $g^{\prime}\left(z_{t}\right)$ can be expressed as

$$
2 g^{\prime}\left(z_{t}\right)=T+\mathrm{i} Q / k
$$

where $k$ is the effective heat conductivity defined as

$$
k=\sqrt{k_{11} k_{22}-k_{12}^{2}} \text {. }
$$

Alternatively we can also regard the quantity $Q / k$ as a conjugate temperature variable, $\tilde{T}$ (Kuo \& Riccardella 1987), and write (2.12) as

$$
2 g^{\prime}\left(z_{t}\right)=T+\mathrm{i} \tilde{T} .
$$

Once the function $g$ in the heat condunction problem is determined, based on an extended Stroh's formalism (Stroh 1958) for anisotropic elasticity, the general solutions of (2.6) can be expressed as (Clements 1973; Wu 1984; Hwu 1990)

$$
\begin{aligned}
u_{i} & =2 \operatorname{Re}\left\{A_{i k} f_{k}\left(z_{k}\right)+c_{i} g\left(z_{t}\right)\right\}, \\
\psi_{i} & =2 \operatorname{Re}\left\{B_{i k} f_{k}\left(z_{k}\right)+d_{i} g\left(z_{t}\right)\right\}, \\
z_{k} & =x_{1}+p_{k} x_{2}, \\
\boldsymbol{A} & =\left\{\boldsymbol{a}_{1}, \boldsymbol{a}_{2}, \boldsymbol{a}_{3}\right\}, \quad \boldsymbol{B}=\left\{\boldsymbol{b}_{1}, \boldsymbol{b}_{2}, \boldsymbol{b}_{3}\right\},
\end{aligned}
$$

where the elastic eigenvectors $\{\boldsymbol{a}, \boldsymbol{b}\}$ and the elastic eigenvalue $\boldsymbol{p}$ are determined by the following eigenvalue problem

$$
\boldsymbol{N} \boldsymbol{\xi}=p \boldsymbol{\xi}, \quad \boldsymbol{N}=\left[\begin{array}{ll}
\boldsymbol{N}_{1} & \boldsymbol{N}_{2} \\
\boldsymbol{N}_{3} & \boldsymbol{N}_{1}^{\mathrm{T}}
\end{array}\right], \quad \boldsymbol{\xi}=\left\{\begin{array}{l}
\boldsymbol{a} \\
\boldsymbol{b}
\end{array}\right\}
$$

Proc. R. Soc. Lond. A (1993) 
where $\boldsymbol{N}_{1}=-\boldsymbol{T}^{-1} \boldsymbol{R}^{\mathrm{T}}, \boldsymbol{N}_{2}=\boldsymbol{T}^{-1}=\boldsymbol{N}_{2}^{\mathrm{T}}, \boldsymbol{N}_{3}=\boldsymbol{R} \boldsymbol{T}^{-1} \boldsymbol{R}^{\mathrm{T}}-\boldsymbol{Q}=\boldsymbol{N}_{3}^{\mathrm{T}}$, with

$$
Q_{i k}=C_{i 1 k 1}, \quad R_{i k}=C_{i 1 k 2}, \quad T_{i k}=C_{i 2 k 2},
$$

the superscript $\mathrm{T}$ stands for the transpose of a matrix; while the vectors $\{\boldsymbol{c}, \boldsymbol{d}\}$ are the solution of

$$
\boldsymbol{N} \boldsymbol{\eta}=\tau \boldsymbol{\eta}+\boldsymbol{\gamma}, \quad \boldsymbol{\gamma}=-\left[\begin{array}{ll}
0 & \boldsymbol{N}_{2} \\
\boldsymbol{I} & \boldsymbol{N}_{1}^{\mathrm{T}}
\end{array}\right]\left\{\begin{array}{l}
\boldsymbol{\beta}_{1} \\
\boldsymbol{\beta}_{2}
\end{array}\right\}, \quad \boldsymbol{\eta}=\left\{\begin{array}{l}
\boldsymbol{c} \\
\boldsymbol{d}
\end{array}\right\},
$$

where $\boldsymbol{\beta}_{1}=\left\{\beta_{i 1}\right\}, \boldsymbol{\beta}_{2}=\left\{\beta_{i 2}\right\}$ and $\boldsymbol{I}$ is the identity matrix. The vector $\boldsymbol{\psi}$ given by $(2.16)$ is the stress function defined such that

$$
\sigma_{i 1}=-\psi_{i, 2}, \quad \sigma_{i 2}=\psi_{i, 1} .
$$

The property of positive definiteness of the elastic constants $C_{i j k l}$ implies that the elastic eigenvalues $p_{k}$ can not be real numbers in (2.19). It must be emphasized that the general solutions of the system (2.6) given by (2.15) and (2.16) break down if the thermal eigenvalue, $\tau$, is equal to one of the elastic eigenvalues, $p$ since in this case (2.21) fails to determine $\boldsymbol{c}$ and $\boldsymbol{d}$. However, for $\tau=p$, in some cases, some information of the solutions can still be derived by making use of the following identities

$$
\begin{gathered}
\boldsymbol{S}=\mathrm{i}\left(2 \boldsymbol{A} \boldsymbol{B}^{\mathrm{T}}-\boldsymbol{I}\right), \quad \boldsymbol{H}=2 \mathrm{i} \boldsymbol{A} \boldsymbol{A}^{\mathrm{T}}, \quad \boldsymbol{L}=-2 \mathrm{i} \boldsymbol{B} \boldsymbol{B}^{\mathrm{T}}, \\
\tilde{\boldsymbol{\gamma}}=-\boldsymbol{L} \boldsymbol{c}+\boldsymbol{S}^{\mathrm{T}} \boldsymbol{d}-\mathrm{i} \boldsymbol{d},
\end{gathered}
$$

where the real matrices $\boldsymbol{S}, \boldsymbol{H}$ and $\boldsymbol{L}$ and vector $\tilde{\boldsymbol{\gamma}}$ can be determined directly from $\boldsymbol{C}$ and $\boldsymbol{\beta}_{1}, \boldsymbol{\beta}_{2}$ (Barnett \& Lothe 1973; Hwu 1990).

\section{Conservation laws}

Let us rewrite (2.15) and (2.16) as

$$
u_{i}=\tilde{u}_{i}+u_{i}^{*}, \quad \psi_{i}=\tilde{\psi}_{i}+\psi_{i}^{*},
$$

where

$$
\begin{array}{rlrl}
\tilde{u}_{i} & =2 \operatorname{Re}\left\{A_{i k} f_{k}\left(z_{k}\right)\right\}, & \tilde{\psi}_{i}=2 \operatorname{Re}\left\{B_{i k} f_{k}\left(z_{k}\right)\right\}, \\
u_{i}^{*} & =2 \operatorname{Re}\left\{c_{i} g\left(z_{t}\right)\right\}, & & \psi_{i}^{*}=2 \operatorname{Re}\left\{d_{i} g\left(z_{t}\right)\right\} .
\end{array}
$$

The displacements $\tilde{u}_{i}$ and $u_{i}^{*}$ basically represent the homogeneous and particular solutions, respectively, of $(2.6)$. In fact the expressions for $\tilde{u}_{i}$ and $\tilde{\psi}_{i}$ are identical in form with the displacement and stress function in Stroh's formalism for isothermal elasticity. Consequently, any conservation law for steady-state thermal elasticity can thus be established from its counterpart for isothermal elasticity. For isothermal anisotropic elasticity, a complete analysis of the first-order conservation laws depending on $\boldsymbol{x}, \boldsymbol{u}$ and the first derivatives of $\boldsymbol{u}$ for the general anisotropic isothermal elasticity has been carried out by Yeh et al. (previous paper) based on Stroh's formalism. It has been shown that, except for the wellknown Betti's law, there are three infinite-dimensional families of conservation laws, each depending on an arbitrary analytic function $F_{k}\left(z_{k}, \varphi_{k}\right)$ of two complex variables, where $z_{k}$ are given by (2.17) and $\tilde{\varphi}_{k}$ are provided by

$$
\begin{aligned}
\varphi_{k}\left(z_{k}\right) & =f_{k}^{\prime}\left(z_{k}\right)=A_{i k} \tilde{\sigma}_{i 2}+B_{i k} \tilde{u}_{i, 1} \\
& =p_{k}^{-1}\left(-A_{i k} \tilde{\sigma}_{i 1}+B_{i k} \tilde{u}_{i, 2}\right) \quad(\text { no sum over } k) .
\end{aligned}
$$

Proc. R. Soc. Lond. A (1993) 
For example, the well-known $J$-integral (Rice 1968) and $M$-integral (Knowles \& Sternberg 1972) for isothermal elasticity can be defined similarly for steady-state linear thermoelasticity as

$$
\begin{gathered}
\tilde{J}=\int\left(\tilde{W} n_{1}-\tilde{t}_{i} \tilde{u}_{i, 1}\right) \mathrm{d} s=\operatorname{Re}\left\{\sum_{k=1}^{3} \int \tilde{\varphi}_{k}^{2} d z_{k}\right\}, \\
\tilde{M}=\int\left(\tilde{W} x_{k} n_{k}-\tilde{t}_{i} \tilde{u}_{i, j} x_{j}\right) \mathrm{d} s=\operatorname{Re}\left\{\sum_{k=1}^{3} \int z_{k} \tilde{\varphi}_{k}^{2} d z_{k}\right\}, \\
\tilde{W}=\frac{1}{2} \tilde{\sigma}_{i j} \tilde{u}_{i, j}, \quad \tilde{t}_{i}=\tilde{\sigma}_{i j} n_{j},
\end{gathered}
$$

in which $\tilde{W}$ and $\tilde{\sigma}_{i}$ are the strain energy density and traction, respectively, corresponding to $\tilde{u}_{i} ; n_{i}$ is the component of the unit outward vector normal to the contour.

We now turn to the thermal stress problem of linear isotropic elasticity for which the elastic and thermal eigenvalues are identical; i.e. $p_{1}=p_{2}=\tau=\mathrm{i}$. As remarked in the previous section, (2.15) and (2.16) are not valid for isotopic materials. However, the decomposition of the actual displacement given by (3.1) as well as the forms of the $\tilde{J}$ and $\tilde{M}$ still holds. In fact, the particular solution $u_{i}^{*}$ can be chosen as the displacement derived from the stress-free thermal strain (Muskhelishvili 1953). Thus $\boldsymbol{c}$ and $\boldsymbol{d}$ are given by

$$
\boldsymbol{c}=\alpha_{1}\left\{\begin{array}{c}
1 \\
-\mathrm{i}
\end{array}\right\}, \quad \boldsymbol{d}=\mathbf{0} .
$$

where $\alpha_{1}=(1+\nu) \alpha$ with $\nu$ being Poisson's ratio and $\alpha$ being the thermal expansion coefficient. With (3.8), the $\tilde{J}$ and $\tilde{M}$ integrals given by (3.5) and (3.6) become

$$
\begin{aligned}
\tilde{J} & =\int\left\{\tilde{W} n_{1}-t_{i} u_{i, 1}+\alpha_{1}\left(t_{1} T+t_{2} \tilde{T}\right)\right\} \mathrm{d} s \\
\tilde{M} & =\int\left\{\tilde{W} x_{k} n_{k}-t_{i} u_{i, j} x_{j}+\alpha_{1} T\left(t_{1} x_{1}+t_{2} x_{2}\right)-\alpha_{1} \tilde{T}\left(t_{1} x_{2}-t_{2} x_{1}\right)\right\} \mathrm{d} s,
\end{aligned}
$$

where $\tilde{T}$ is the conjugate temperature defined by (2.14). The expressions of $\tilde{J}$ and $\tilde{M}$ integrals in (3.9) and (3.10) agree with those derived by Kuo \& Riccardella (1987).

\section{Applications to fracture mechanics}

Consider a thermoelastic solid containing a finite crack $\left|x_{1}\right| \leq a$. The crack faces are assumed free of tractions mechanically and subject to either heat flux or temperature boundary conditions thermally. In the following discussion superscripts + and - are attached to the quantities evaluated on the upper and lower crack faces, respectively, and $[(\cdots)]=(\cdots)^{+}-(\cdots)^{-}$is used to denote the jump across the crack of the quantity in the bracket. Let $\Gamma$ be a contour starting from $x_{1}=c^{-}$on the lower crack face, terminating at $x_{1}=c^{+}$on the upper crack face and encircling the crack tip at $x_{1}=a$. The fact that the $\tilde{J}$ integral given by (3.5) 
is zero for closed integration paths yields

$$
\tilde{J}_{\Gamma}+\int_{c}^{a}\left[\sigma_{2 i}^{*} \tilde{u}_{i, 1}\right] \mathrm{d} x_{1}=\tilde{J}_{0},
$$

where $\tilde{J}_{\Gamma}$ and $\Gamma_{0}$ are the values of the $\tilde{J}$ integral integrated along $\Gamma$ and a contour infinitely close to the crack tip respectively; $\sigma_{i 2}^{*}$ is the stress calculated from $\psi^{*}$ given by (3.1). In deriving (4.1), the traction-free conditions $t_{i}=0$ have been used. If we assume that the temperature is finite at the crack tip, the thermal stresses $\sigma_{i j}^{*}$ associated with the displacement $\boldsymbol{u}^{*}$ are also finite there. Thus the crack-tip fields for $\tilde{\boldsymbol{u}}$ are in the same form as those under isothermal condition and $\tilde{J}_{0}$ is equal to the energy release rate associated with translation of the crack tip given by (Barnett \& Asaro 1972)

$$
\tilde{J}_{0}=\frac{1}{2} K_{p} L_{p q}^{-1} K_{q},
$$

where $\boldsymbol{L}$ is given by (2.23) and $K_{p}$ are the stress intensity factors defined by

$$
K_{p}=\left.\lim _{r \rightarrow 0} \sqrt{2 \pi r} \sigma_{p 2}\right|_{\theta=0},
$$

with $(r, \theta)$ being the polar coordinates with origin at the crack tip. Equation (4.1) is similar in form as that for isothermal elasticity except the presence of the second contour integral along the crack line. The extra term vanishes only for isotropic materials for which $\boldsymbol{\sigma}^{*}=\mathbf{0}$. Similar discussion for the $\tilde{M}$-integral gives

$$
\tilde{M}_{\Gamma}+\int_{c}^{a} x_{1}\left[\sigma_{2 i}^{*} \tilde{u}_{i, 1}\right] \mathrm{d} x_{1}=a \tilde{J}_{0} .
$$

Equations (4.1) and (4.4) can be used to calculate the energy release rates. However, in fracture mechanics one is interested in not only the energy release rates but the separate stress intensity factors. In most problems for anisotropic thermal elastic materials, all three intensity factors are usually non-zero and can not be determined by the energy release rate alone. In linear two-dimensional anisotropic isothermal elasticity, path-independent integrals directly related to the stress intensity factors of a straight crack of finite length are derived by $\mathrm{Wu}$ (1989). With similar arguments as those for the $\tilde{J}$ integral, the path-independent integrals of Wu can also be extended to include steady-state thermal effects as

$$
\begin{aligned}
& K_{p}=\int_{\Gamma}\left(\tilde{G}_{p i} \frac{\partial \tilde{u}_{i}}{\partial s}-\tilde{H}_{p i} \tilde{t}_{i}\right) \mathrm{d} s \\
& +\frac{1}{2 \sqrt{\pi a}} \int_{-a}^{a}\left(\operatorname{Re}\{\boldsymbol{d}\}\left(T^{+}+T^{-}\right)-k^{-1} \operatorname{Im}\{\boldsymbol{d}\}\left(Q^{+}+Q^{-}\right)\right) \sqrt{\frac{a+x_{1}}{a-x_{1}}} \mathrm{~d} x_{1},
\end{aligned}
$$

where Im denotes the imaginary part and

$$
\begin{aligned}
& \tilde{G}_{p i}\left(z_{k}\right)=\frac{1}{\sqrt{\pi a}} \operatorname{Im}\left(B_{p k} B_{i k} \sqrt{\frac{z_{k}+a}{z_{k}-a}}\right), \\
& \tilde{H}_{p i}\left(z_{k}\right)=\frac{1}{\sqrt{\pi a}} \operatorname{Im}\left(B_{p k} A_{i k} \sqrt{\frac{z_{k}+a}{z_{k}-a}}\right) .
\end{aligned}
$$

Proc. R. Soc. Lond. A (1993) 
Equation (4.5) is for the stress intensity factors associated with the tip at $x_{1}=a$. The contour $\Gamma$ is arbitrary provided that it encloses the crack tip at $x_{1}=a$ and possibly the tip at $x_{1}=-a$ but no other elastic or thermal singularities. The expressions for the tip at $x_{1}=-a$ can be derived similarly. The expressions given by (4.7) for $\tilde{\boldsymbol{G}}$ and $\tilde{\boldsymbol{H}}$ are for anisotropic materials. The corresponding expressions for isotropic materials can be obtained by regarding them as slightly orthotropic. Using (4.5) to compute the stress intensity factors only requires the gradients of $\tilde{\boldsymbol{u}}$ and the tractions $\tilde{\boldsymbol{t}}$ on an arbitrary contour enclosing the crack in addition to $T$ and $Q$ on the crack faces.

As an application of (4.5), let the medium containing the crack be unbounded and the stresses in the medium be solely due to the thermal boundary conditions on the crack faces. In this case, we can stretch the contour to infinity and denote it as $\Gamma_{\infty}$. By letting $z_{\alpha} \rightarrow \infty$ in $\tilde{\boldsymbol{G}}$ and $\tilde{\boldsymbol{H}}$ of (4.7), (4.5) becomes

$$
\begin{aligned}
K_{p}= & \tilde{G}_{p i}(\infty) \int_{\Gamma_{\infty}} \frac{\partial u_{i}}{\partial s} \mathrm{~d} s-\tilde{H}_{p i}(\infty) \int_{\Gamma_{\infty}} t_{i} \mathrm{~d} s \\
& -\tilde{G}_{p i}(\infty) \int_{\Gamma_{\infty}} \frac{\partial u_{i}^{*}}{\partial s} \mathrm{~d} s+\tilde{H}_{p i}(\infty) \int_{\Gamma_{\infty}} t_{i}^{*} \mathrm{~d} s \\
& +\frac{1}{2 \sqrt{\pi a}} \int_{-a}^{a}\left(\operatorname{Re}\left\{\boldsymbol{d}\left(T^{+}+T^{-}\right)\right\}-k^{-1} \operatorname{Im}\{\boldsymbol{d}\}\left(Q^{+}+Q^{-}\right)\right) \sqrt{\frac{a+x_{1}}{a-x_{1}}} \mathrm{~d} x_{1},
\end{aligned}
$$

in which

$$
\tilde{\boldsymbol{G}}(\infty)=\frac{1}{2 \sqrt{\pi a}} \boldsymbol{L}, \quad \tilde{\boldsymbol{H}}(\infty)=\frac{-1}{2 \sqrt{\pi a}} \boldsymbol{S}^{\mathrm{T}},
$$

where $\boldsymbol{L}$ and $\boldsymbol{S}$ are given by (2.23). As there are no body forces or dislocations present in the medium, we have

$$
\int_{\Gamma_{\infty}} \frac{\partial u_{i}}{\partial s} \mathrm{~d} s=0, \quad \int_{\Gamma_{\infty}} t_{i} \mathrm{~d} s=0 .
$$

With (4.10) and (3.3), (4.8) is simplified as

$$
\boldsymbol{K}=\frac{1}{\sqrt{\pi a}} \operatorname{Re}\left\{\left(-\boldsymbol{L} c+\boldsymbol{S}^{\mathrm{T}} \boldsymbol{d}\right) \int_{\Gamma_{\infty}} g^{\prime}\left(z_{t}\right) \mathrm{d} z_{t}+\boldsymbol{d} \int_{-a}^{a}\left(g^{\prime+}+g^{\prime-}\right) \sqrt{\frac{a+x_{1}}{a-x_{1}}} \mathrm{~d} x_{1}\right\}
$$

Note that (4.11) is expressed in terms of the analytic function $g^{\prime}$ only and thus can be calculated once the steady state heat conduction problem is solved.

Consider the case where the heat flux, $q_{2}$, normal to the crack line is prescribed as $q_{2}=q_{o}\left(x_{1}\right)$ on the crack faces. Use of the standard technique of analytic continuation leads to the following Hilbert problem:

$$
g^{\prime \prime+}+g^{\prime \prime-}=\mathrm{i} q_{o} / k
$$

on $\left|x_{1}\right| \leq a$. If $g^{\prime \prime}$ is assumed to approach zero as $z_{t} \rightarrow \infty$ and $g^{\prime}$ is assumed single valued except on the crack line, the solution to (4.12) is given by

$$
g^{\prime \prime}\left(z_{t}\right)=\frac{\mathrm{i}}{2 \pi \mathrm{k}} \frac{1}{\sqrt{ }\left(z_{t}^{2}-a^{2}\right)} \int_{-a}^{a} \frac{q_{o} \sqrt{ }\left(a^{2}-s^{2}\right)}{s-z_{t}} \mathrm{~d} s .
$$

Proc. R. Soc. Lond. A (1993) 
From (4.13), it can be shown that

$$
\int_{\Gamma_{\infty}} g^{\prime}\left(z_{t}\right) \mathrm{d} z_{t}=-k^{-1} \int_{-a}^{a} q_{o} \sqrt{a^{2}-s^{2}} \mathrm{~d} s
$$

where $g^{\prime}$ is assumed to approach zero as $z_{t}$ approaches infinity. Furthermore, integrating (4.12) with respect to $x_{1}$, we have

$$
g^{\prime}(s)^{+}+g^{\prime}(s)^{-}=(\mathrm{i} / k) Q_{o}(s)
$$

where $Q_{o}(s)=\int q_{o}(s) \mathrm{d} s$. Substituting (4.14) and (4.15) into (4.11), we obtain

$$
\boldsymbol{K}=\frac{1}{k \sqrt{\pi a}} \operatorname{Re}\left\{\left(\boldsymbol{L} c-\boldsymbol{S}^{\mathrm{T}} \boldsymbol{d}\right) \int_{-a}^{a} q_{o} \sqrt{a^{2}-s^{2}} \mathrm{~d} s+\mathrm{i} \boldsymbol{d} \int_{-a}^{a} Q_{o} \sqrt{\frac{a+s}{a-s}} \mathrm{~d} s\right\} .
$$

Since

$$
\int_{-a}^{a} Q_{o} \sqrt{\frac{a+s}{a-s}} \mathrm{~d} s=\int_{-a}^{a} q_{o} \sqrt{a^{2}-s^{2}} \mathrm{~d} s+a \int_{-a}^{a} \frac{Q_{o}}{\sqrt{a^{2}-s^{2}}} \mathrm{~d} s
$$

(4.16) can also be expressed as

$$
\boldsymbol{K}=-\frac{1}{k \sqrt{\pi a}}\left\{\operatorname{Re}\{\tilde{\boldsymbol{\gamma}}\} \int_{-a}^{a} q_{o} \sqrt{a^{2}-s^{2}} \mathrm{~d} s+a \operatorname{Im}\{\boldsymbol{d}\} \int_{-a}^{a} \frac{Q_{o}}{\sqrt{a^{2}-s^{2}}} \mathrm{~d} s\right\},
$$

where $\tilde{\gamma}$ is given by (2.24). It is remarkable that the stress intesnity factors are affected by thermal conductivities only through the effective conductivity given by (2.13). Moreover, anisotropy of materials basically alters the direction of the vector $\boldsymbol{K}$ through $\operatorname{Re}\{\tilde{\boldsymbol{\gamma}}\}$ and $\operatorname{Im}\{\boldsymbol{d}\}$. If $q_{o}$ is an even function, the second term on the right-hand side of (4.18) vanishes and $\boldsymbol{k}$ is simply given by

$$
\boldsymbol{K}=-\frac{2}{k \sqrt{\pi a}} \operatorname{Re}\{\tilde{\gamma}\} \int_{0}^{a} q_{o} \sqrt{a^{2}-s^{2}} \mathrm{~d} s .
$$

As mentioned previously $\tilde{\gamma}$ can be directly computed from the elastic constants and thermal conductivity so (4.19) is valid for either degenerate or non-degenerate materials. The result for uniform $q_{o}$ calculated by (4.19) agrees with that reported by Hwu (1990), which is obtained by solving the full boundary-value problem. On the other hand, if $q_{o}$ is an odd function of $s$, the first term on the right-hand side of (4.18) vanishes and $\boldsymbol{K}$ is given by

$$
\boldsymbol{K}=-\frac{2}{k} \sqrt{\frac{a}{\pi}} \operatorname{Im}\{\boldsymbol{d}\} \int_{0}^{a} \frac{Q_{o}}{\sqrt{a^{2}-s^{2}}} \mathrm{~d} s .
$$

In particular, for isotropic materials, $\boldsymbol{d}=\mathbf{0}$, and the stress intensity factors given by (4.20) are zero. The stress intensity factors due to temperature $T_{o}$ prescribed on the upper and lower crack faces can be derived by a similar procedure discussed above. The result is given by

$$
\boldsymbol{K}=-\frac{1}{\sqrt{\pi a}}\left\{\operatorname{Im}\{\tilde{\boldsymbol{\gamma}}\} \int_{-a}^{a} \frac{T_{o} s}{\sqrt{a^{2}-s^{2}}} \mathrm{~d} s-a \operatorname{Re}\{\boldsymbol{d}\} \int_{-a}^{a} \frac{T_{o}}{\sqrt{a^{2}-s^{2}}} \mathrm{~d} s\right\} .
$$

Similar to (4.18), the first term on the right-hand side of (4.11) is zero if $T_{o}$ is an even function while the second term is zero if $T_{o}$ is odd. Equation (4.21) implies 
that for isotropic materials $\boldsymbol{K}=\mathbf{0}$ with a symmetric distribution of temperature on the crack faces. It should be pointed out that in deriving (4.21), $g^{\prime}$ as well as $g^{\prime \prime}$ is assumed to vanish at infinity so that the case where $T_{o}$ is uniform is precluded since the corresponding solution is either uniform in the entire body or becomes unbounded at infinity (Clements 1973).

\section{Conclusions}

A class of conservation laws for anisotropic steady-state thermoelasticity has been developed. The proposed conservation laws lead to path-independent integrals that can be applied numerically to determine the energy release rates or stress intensity factors for thermoelastic crack problems with finite geometries. This application to thermal elastic fracture mechanics requires in general the values of the displacements and tractions as well as temperature and heat function on any convenient contour and possibly on the crack faces. The path-independent integrals can also be used analytically for problems concerning infinite media. Indeed, using one of the path-independent integrals, analytic expressions for the stress intensity factors are obtained for a crack subject to arbitrary heat flux or temperature conditions on the crack faces.

\section{References}

Barnett, D. M. \& Asaro, R. J. 1972 The fracture mechanics of slit-like cracks in anisotropic elastic media. J. Mech. Phys. Solids 20, 353-366.

Barnett, D. M. \& Lothe, J. 1973 Synthesis of the sextic and the integral formalism for dislocation, Green's function and surface waves in anisotropic elastic solids. Phys. Norv. 7, 13-19.

Clements, D. L. 1973 Thermal stress in an anisotropic elastic half-space. SIAM J. appl. Math. 24, 332-337.

Gurtin, M. E. 1979 On a path-independent integral for thermoelasticity. Int. J. Fracture 15, R169-R170.

Hwu, C. 1990 Thermal stress in an anisotropic plate disturbed by an insulated elliptic hole or crack. ASME J. appl. Mech. 57, 916-922.

Kishimoto, K., Aoki, S. \& Sakata, M. 1980 On the path-independent integral- $\hat{J}$. J. Engng Fracture Mech. 13, 841-850.

Knowles, J. K. \& Sternberg, E. 1972 On a class of conservation laws in linearized and finite elastostatics. Arch. ration. Mech. Analysis 44, 187-211.

Kuo, A. N.-Yu \& Riccardella, P.C. 1987 Path-independent line integrals for steady-state, twodimensional thermoelasticity. Int. J. Fracture 35, 71-79.

Lekhnitskii, S. G. 1963 Theory of elasticity of an anisotropic body. San Francisco: Holden-Day.

Muskhelishvili, N. I. 1953 Some basic problems of the mathematical theory of elasticity. Groningen, The Netherlands: P. Noordhoff.

Nowacki, W. 1962 Thermoelasticity. Reading: Addison Wesley.

Rice, J. R. 1968 A path-independent integral and the approximate analysis of strain concentration by notches and cracks. ASME J. appl. Mech. 35, 379-386.

Stroh, A. N. 1958 Dislocations and cracks in anisotropic elasticity. Phil. Mag. 3, 625-646.

Wilson, W. K. \& Yu, L.-W. 1979 The use of the $J$-integral in thermal stress crack problems. . Int. J. Fracture Mech. 15, 377-387.

Wu, C. H. 1984 Plane anisotropic thermoelasticity. ASME J. appl. Mech. 51, 724-726.

Wu, K.-C. 1989 Representations of stress intensity factors by path-independent integrals. ASME J. appl. Mech. 56, 780-785.

Received 15 December 1992; accepted 19 February 1993

Proc. R. Soc. Lond. A (1993) 